\title{
Program/Erase Characteristics of Amorphous Gallium Indium Zinc Oxide Nonvolatile Memory
}

\author{
Huaxiang Yin, Member, IEEE, Sunil Kim, Hyuck Lim, Yosep Min, Chang Jung Kim, Ihun Song, Jaechul Park, \\ Sang-Wook Kim, Alexander Tikhonovsky, Jaewoong Hyun, and Youngsoo Park
}

\begin{abstract}
Currently, both high-density 3-D stacking nonvolatile (NV) memory and embedded NV memory in advanced systems on panel (SOPs) urgently demand the assistance of new and functional transition metal-oxide materials. This is to overcome serious fabrication issues encountered in the use of conventional $\mathrm{Si}$ or poly-crystalline Si materials, as well as to increase storage density with lower process cost. This paper reports the fully functional NV memory structure operated by an ionic amorphous oxide semiconductor with a wide energy band gap $(>3.0 \mathrm{eV})$ in a $\mathrm{Ga}_{2} \mathrm{O}_{3}-\mathrm{In}_{2} \mathrm{O}_{3}-\mathrm{ZnO}$ (GIZO) system under low process temperature $\left(<400{ }^{\circ} \mathrm{C}\right)$ while being combined with various metal-oxide materials of $\mathrm{Al}_{2} \mathrm{O}_{3}, \mathrm{GIZO}$, and $\mathrm{Al}_{2} \mathrm{O}_{3}$ as the electron charge's tunneling, storage, and blocking layers, respectively. The different methods of memory programs and, especially, the unique erase characteristics caused by a much wider band gap than $\mathrm{Si}$ were intensively being investigated, and as a result, excellent electrical results of a large program/erase window over $3.8 \mathrm{~V}$ at a pulse time of $10 \mathrm{~ms}$ are achieved.
\end{abstract}

Index Terms-Amorphous, gallium indium zinc oxide (GIZO), memory, nonvolatile (NV), oxide, program/erase (P/E), thin-film transistor (TFT).

\section{INTRODUCTION}

C URRENTLY, new types of functional transition metaloxide materials are attracting great interest in new types of memory device technologies [1]-[3]. This is because of the material advantages over the usual applied silicon $(\mathrm{Si})$ or other conventional materials in terms of fundamental innovations to the conventional fabrication method, subsequently enhancing electronic products' performance and increasing circuit density. Meanwhile, as the device dimension scale into the nanoscale range, physical and electrical characteristics, as well as the reliability scaling challenges of the nonvolatile (NV) memory, become more serious [4]. A series of new technologies, such as the high- $k$ material in storage cells and 3-D device structures and the low- $k$ material for adjacent cell filling, has been de-

Manuscript received March 18, 2008. This work was supported by Samsung Advanced Institute of Technology. The review of this paper was arranged by Editor V. R. Rao.

H. Yin, S. Kim, H. Lim, C. J. Kim, I. Song, J. Park, S.-W. Kim, and Y. Park are with the Semiconductor Device Laboratory, Samsung Advanced Institute of Technology, Yongin 449-712, Korea (e-mail: huaxiang.yin@ samsung.com).

Y. Min was with the Semiconductor Device Laboratory, Samsung Advanced Institute of Technology, Yongin 449-712, Korea.

A. Tikhonovsky was with the AE Center, Samsung Advanced Institute of Technology, Yongin 446-712, Korea. He is now with the Samsung SDI Co., Ltd., Suwon 442-390, Korea.

J. Hyun was with the Semiconductor Device Laboratory, Samsung Advanced Institute of Technology, Yongin 449-712, Korea. He is now with Harvard University, Cambridge, MA 02138-3800 USA.

Color versions of one or more of the figures in this paper are available online at http://ieeexplore.ieee.org.

Digital Object Identifier 10.1109/TED.2008.926727 veloped to overcome those issues. On the other hand, layer-bylayer stacking devices on one substrate under low process temperature are considered as one of the most effective solutions to fundamentally relax these limits. Some types of multilayer resistive RAMs that are composed of transition metal-oxides, such as $\mathrm{NiO}$ or $\mathrm{SrTiO} 3$, and $\left[\mathrm{Pr}_{1-x} \mathrm{Ca}_{x} \mathrm{MnO}_{3}\right.$, (PraseodymiumCalcium-Manganese-Oxide)] [5]-[7] resistors and diodes have been explored for next-generation NV memory. Additionally, the two-layer NAND Flash memory by a highly stackable polycrystalline Si thin-film transistor (TFT) prototype has been presented [8]. However, most of these materials are expected to show diverse material and device characteristics between the intra- and intergrain due to the mixed-crystal structures. Therefore, it always results in nonuniform threshold voltage distribution in the electrical devices [9], which makes it difficult to apply them to mature electronic products, especially with the stringent uniformity requirement in the modern electronic industry.

One of the new transition metal-oxide materials, the ionic amorphous oxide semiconductor (IAOS) with a wide energy band gap $\left(>3.0 \mathrm{eV}\right.$ ) in a $\mathrm{Ga}_{2} \mathrm{O}_{3}-\mathrm{In}_{2} \mathrm{O}_{3}-\mathrm{ZnO}$ system has been developed for future advanced transparent-display or flexibledisplay applications [10], [11]. This material, namely, amorphous gallium indium zinc oxide (a-GIZO), demonstrates an electron mobility that is one magnitude higher $\left(\geq 10 \mathrm{~cm}^{2} / \mathrm{V} \cdot \mathrm{s}\right)$ than that of a-Si:H, even with a similar amorphous state, which is attributed to the different material structure and electron conduction mechanics. A number of applications, even with the simple inverter or oscillator circuit based on this material [12]-[14] for the value-added application in the system on panel (SOP), have been explored. For a long time, to support the most advanced SOP product incorporating the CPU and memory circuit, low-temperature poly-crystalline TFTs by various advanced crystallization methods have extensively been developed [15], [16]. However, these techniques always suffer the similar issue of nonuniform electrical characteristics in 3-D stacking memory.

In this paper, for the first time, the material structure and electrical characteristics of an a-GIZO TFT with a fully functional $\mathrm{NV}$ memory structure for applications of 3-D stacking memory or embedded memory in advanced SOP are demonstrated. The memory electrical characteristics, especially for the unique memory erase characteristics due to the special charge injection mechanics for the standard memory structure on a special widegap oxide semiconductor, were intensively investigated, and finally, a good program/erase $(\mathrm{P} / \mathrm{E})$ window and speed were achieved. 


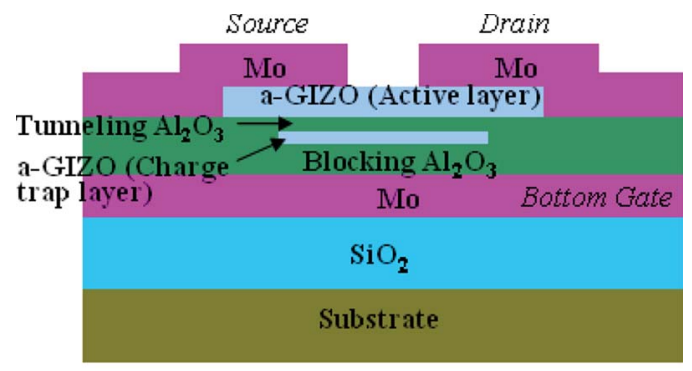

(a)

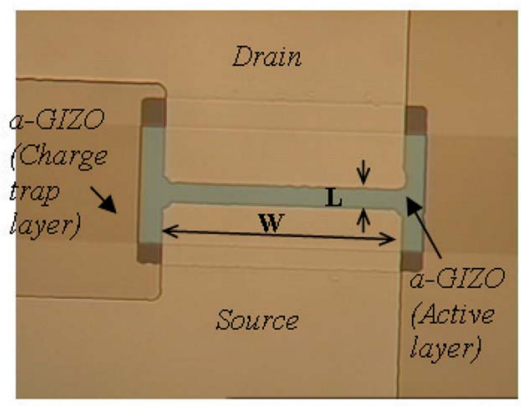

(b)

Fig. 1. (a) Cross-sectional view and (b) top view of the wide-band-gap a-GIZO TFT NV memory using the a-GIZO and $\mathrm{Al}_{2} \mathrm{O}_{3}$ as charge trap layer and gate insulators, respectively.

\section{FABRicAtion PROCESS}

The schematic cross-sectional structure of an a-GIZO TFT with an NV memory structure is shown in Fig. 1, where the top-view optical image by microscope is also included. The device has an inverted staggered bottom-gate structure. At first, a 100-nm metal Mo for the bottom-gate electrode was deposited by magnetic RF sputtering on a 6-in Si wafer covered with 500-nm-thick oxide. The sandwich charge storage structure consisted of three layers of 35-nm atom-layer-deposition (ALD) aluminum oxide $\left(\mathrm{Al}_{2} \mathrm{O}_{3}\right), 20-\mathrm{nm}$ a-GIZO, and 10-nm ALD $\mathrm{Al}_{2} \mathrm{O}_{3}$, which were formed with the following steps for NV memory application. In this structure, the a-GIZO was deposited by magnetic RF sputtering at room temperature and formed with the floating-gate-type pattern by a standard lithograph and wet etch (diluted hydrofluoric acid) process before the second time deposition of the $\mathrm{Al}_{2} \mathrm{O}_{3}$. Later, another 70-nmthick a-GIZO is deposited by sputtering and formed by a similar lithograph and etch process for the TFT active layer before the final formation of source/drain (S/D) electrodes by Mo. The highest temperature during this process was less than $400{ }^{\circ} \mathrm{C}$.

During the a-GIZO sputtering process, the target of GIZO $\left(\mathrm{Ga}_{2} \mathrm{O}_{3}: \mathrm{In}_{2} \mathrm{O}_{3}: \mathrm{ZnO}=1: 1: 1\right.$, or $\mathrm{Ga}:$ In $\left.: \mathrm{Zn}=2: 2: 1\right)$ with the source gas $\mathrm{Ar} / \mathrm{O}_{2}=19 / 1$ is applied to keep the electron concentration below $5 \times 10^{19} \mathrm{~cm}^{-3}$ [10], [11] to form an appropriate semiconducting material. A number of other amorphous ionic oxides, such as a-IZO or a-IO, have higher carrier mobility; however, it is very difficult to keep the electron carrier concentration below $1 \times 10^{20} \mathrm{~cm}^{-3}$ and be more like a conductor, and the devices with these materials show very high leakage.

The deposited a-GIZO shows a homogeneous amorphous state confirmed by the X-ray diffraction data presented in a previous publication [17]. Even as the process temperature increases to $600{ }^{\circ} \mathrm{C}$, the film has no clear sharp peak observed and has, in fact, kept its amorphous state. The cross-sectional view of the transmission electron microscopy (TEM) image, high-resolution TEM (HRTEM) image, and secondary ion mass spectrometry (SIMS) depth profile analysis data is shown in Fig. 2(a)-(c), which demonstrates detailed information of the materials and the device structure. The HRTEM image of the charge trapping and active layer with a larger image resolution indicates that the GIZO layers still keep their amorphous states after a series of device fabrication steps.

\section{RESULT AND DISCUSSION}

Fig. 3 shows the representative drain-current/gate-voltage $\left(I_{\mathrm{ds}}-V_{\mathrm{gs}}\right)$ transfer characteristics of an n-type a-GIZO TFT with an NV memory structure under $V_{\mathrm{ds}}=0.1 \mathrm{~V}$ and $1.1 \mathrm{~V}$ without any postannealing. (Normally, a 1-h annealing in a $350{ }^{\circ} \mathrm{C}$ furnace with pure $\mathrm{N}_{2}$ is necessary.) The geometric size of all devices studied in this paper is fixed with a channel width/channel length of $(W / L)=50 / 4 \mu \mathrm{m}$. In this figure, the TFT's threshold voltage $V_{\mathrm{th}}$ is defined as the gate voltage inducing a drain current equal to $W \times 1 \mathrm{nA} / \mu \mathrm{m}$ and equal to $0.7 \mathrm{~V}$ at $V_{\mathrm{ds}}=1.1 \mathrm{~V}$. The saturation current is $0.52 \mu \mathrm{A} / \mu \mathrm{m}$ at $V_{\mathrm{ds}}=0.1 \mathrm{~V}$, and the estimated electron carrier field-effect mobility $\mu_{\mathrm{FE}}$ calculated by the traditional one-order approximation definition equation [18] is $10.3 \mathrm{~cm}^{2} / \mathrm{V} \cdot \mathrm{s}$. Moreover, the on-off current ratio $I_{\mathrm{ON}} / I_{\mathrm{OFF}}$ is greater than $5 \times 10^{6}$, and the subthreshold slope is $207 \mathrm{mV} / \mathrm{dec}$. The measured direct current performance merits are much better than those of a-Si:H TFT and are able to arrive at the basic device requirement for operating circuits in an SOP.

The typical programmed and erased $I_{\mathrm{ds}}-V_{\mathrm{gs}}$ curves of the a-GIZO TFT NV memory without postannealing are shown in Fig. 4. In this figure, clear positive and negative shiftings of the $I_{\mathrm{ds}}-V_{\mathrm{gs}}$ curves due to the channel hot electron (CHE) program mode $\left(V_{\mathrm{gs}}=+12 \mathrm{~V}, V_{\mathrm{ds}}=+8\right.$ or $\left.9 \mathrm{~V}\right)$ and one kind of bandto-band tunneling-induced hot hole injection (BB-HHI) erase modes $\left(V_{\mathrm{gs}}=+12 \mathrm{~V}, V_{\mathrm{ds}}=+10\right.$ or $\left.11 \mathrm{~V}\right)$ are observed, respectively [19], [20]. The detailed mechanics of these program and special erase behaviors will be discussed later.

Except for the CHE/BB-HHI program, the direct $+\mathrm{F}-\mathrm{N}$ mode $\left(V_{\mathrm{gs}}>0 \mathrm{~V}\right)$ and $-\mathrm{F}-\mathrm{N}$ mode $\left(V_{\mathrm{gs}}<0 \mathrm{~V}\right)$ on this structure are also investigated while the S/D are grounded. The measured results, together with the comparisons of CHE/BB-HHI, are summarized in Fig. 5, which employ the shifted threshold voltages $\left(\Delta V_{\mathrm{th}}\right)$ to qualify the specific $\mathrm{P} / \mathrm{E}$ characteristics of the devices under different bias conditions. In this figure, $+\mathrm{F}-\mathrm{N}$ properly programs the device like $\mathrm{CHE}$. At a time of $10 \mathrm{~ms}$, the device with both $+\mathrm{F}-\mathrm{N}$ and $\mathrm{CHE}$ modes achieved program window $\Delta V_{\mathrm{th}}>4.0 \mathrm{~V}$. However, $-\mathrm{F}-\mathrm{N}$, which is normally used for an erasing device, provokes very small negative $\Delta V_{\mathrm{th}}$ in the a-GIZO TFT NV memory. With $V_{\mathrm{gs}}=-14 \mathrm{~V}$ at time $=10 \mathrm{~ms}, V_{\mathrm{th}}$ shifted by only $-0.50 \mathrm{~V}$ is observed, which is much less than the positively shifted $V_{\mathrm{th}}$ under counterpart program conditions.

The possible reason for this phenomena is that a-GIZO is a natural n-type semiconductor following the ionic oxide 


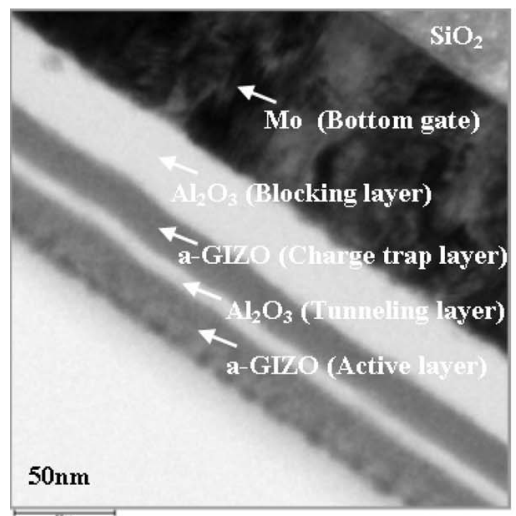

(a)

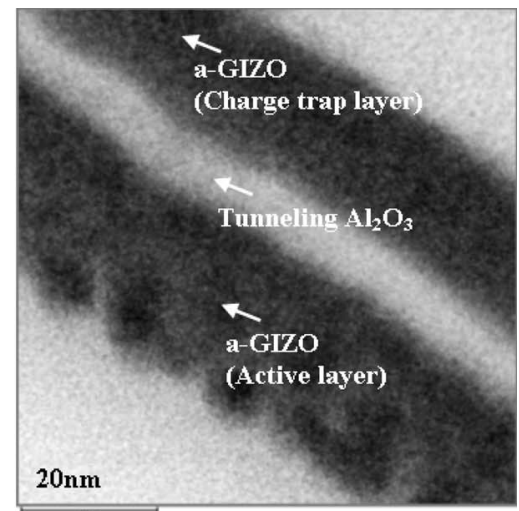

(b)

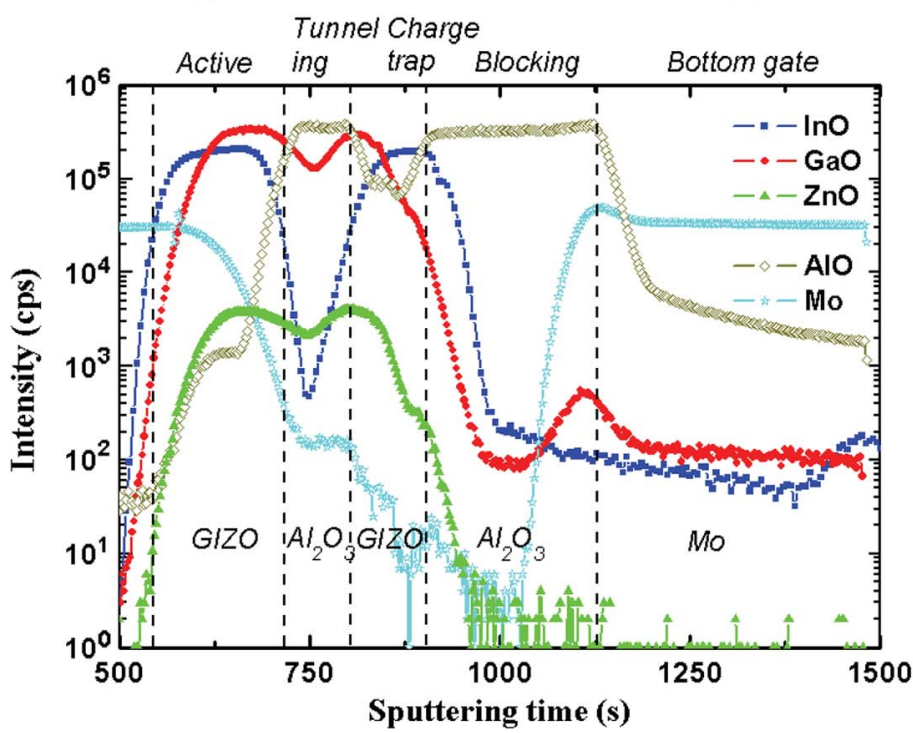

(c)

Fig. 2. (a) TEM image, (b) HRTEM image, and (c) SIMS depth profile analysis data of the a-GIZO TFT NV memory using $10-\mathrm{nm}^{\mathrm{Al}} \mathrm{O}_{2} \mathrm{O}_{3}, 20$-nm a-GIZO, and $35-\mathrm{nm} \mathrm{Al}_{2} \mathrm{O}_{3}$ as the charge tunneling layer, trap layer, and blocking layer, respectively.

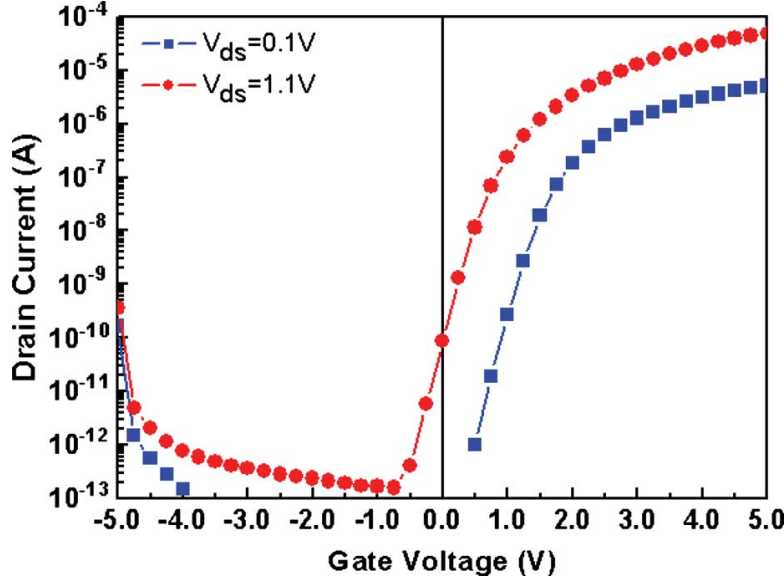

Fig. 3. TFT transfer characteristics of the a-GIZO TFT NV memory before postannealing with channel width/channel length $W / L=50 / 4 \mu \mathrm{m}$.

semiconductor conductance mechanics [11], which is much different from the silicon that is easily doped by $V$ - or III-column ions and turns into an n-type or a p-type semiconductor, respectively. For a-GIZO, there are only electrons that

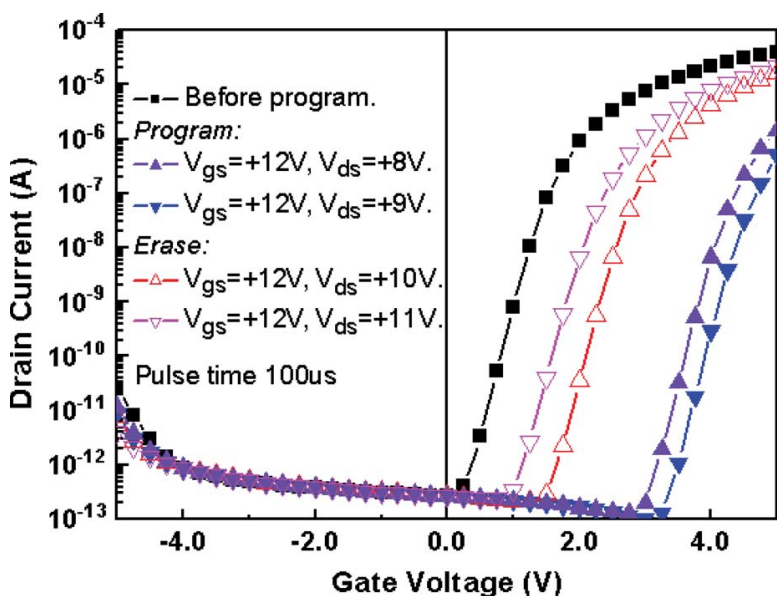

Fig. 4. Typical programmed and erased $I_{\mathrm{ds}}-V_{\mathrm{gs}}$ curves of the a-GIZO TFT NV memory under different stressing conditions.

move around the outmost s-orbital of the neighboring indium or zinc cations and induce the n-type carriers in the device channel to take in the charge transfer through the conduction band minimum in the energy band gap. Since there is no effective 


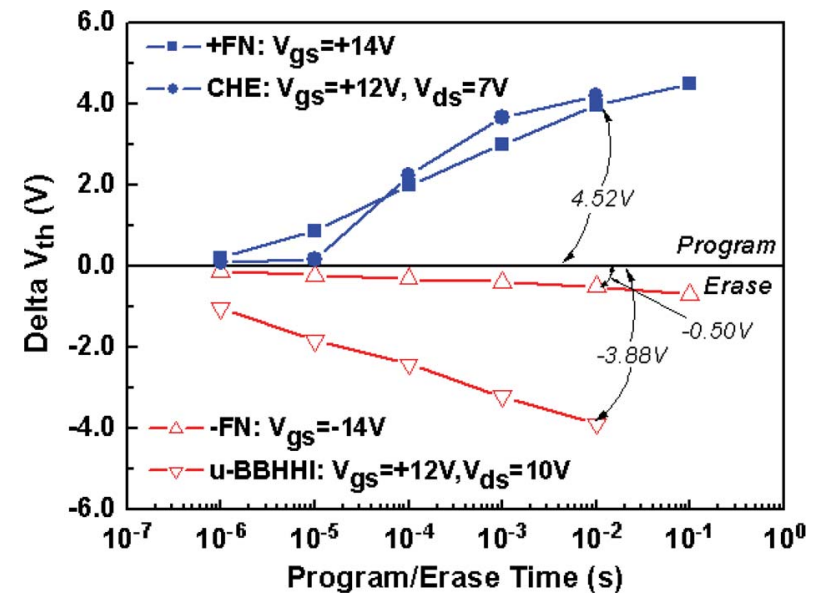

Fig. 5. Summary of the a-GIZO TFT NV memory P/E results by different methods.

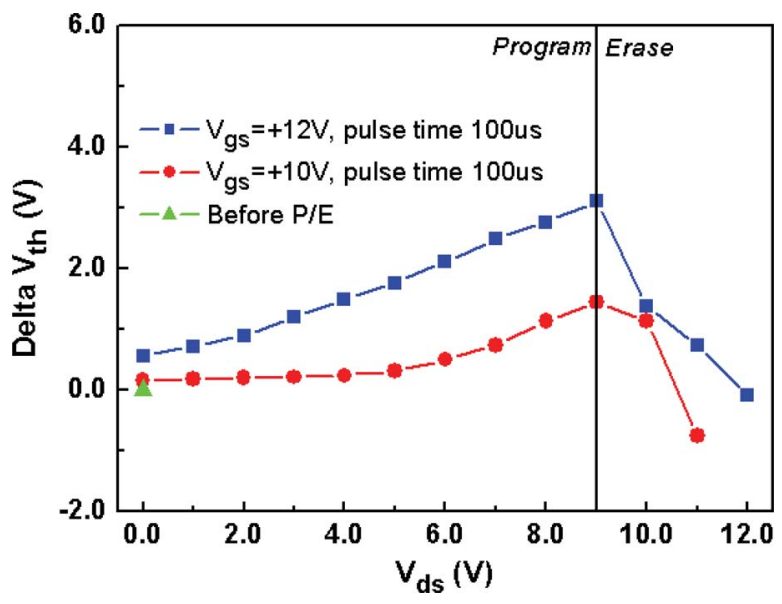

Fig. 6. Shifted $V_{\text {th }}$ (programmed $V_{\mathrm{th}}$-fresh $V_{\mathrm{th}}$ ) as a function of different $V_{\mathrm{ds}}$ 's in the a-GIZO TFT NV memory.

conductance method available for majority electrons in a-GIZO moving in one direction under an externally applied electrical field, which induces the p-type carriers in the device channel to take in the positive charges at a relatively low-energy-level valance band maximum, the hole carrier is difficult to be achieved in all IAOS [11]. Due to this fact, very few holes are available for device erasing when the gate is negatively biased, and a very small negative $\Delta V_{\mathrm{th}}$ under $-\mathrm{F}-\mathrm{N}$ was observed. In contrast, a large positive $\Delta V_{\mathrm{th}}$ is still available through a proper program method with electron injection. As a result, this is one of the disadvantages of the a-GIZO TFT NV memory compared with the conventional Si MOSFET NV memory, which constantly demonstrates similar positive and negative $\Delta V_{\text {th }}$ under the $+\mathrm{F}-\mathrm{N}$ and $-\mathrm{F}-\mathrm{N}$ modes, respectively.

Nevertheless, in Fig. 5, it needs to point out that the erase window by the HHI mode is much larger than that by $-\mathrm{F}-\mathrm{N}$ and nearly symmetrical to the counterpart program window by CHE. With $V_{\mathrm{gs}}=+12 \mathrm{~V}$ and $V_{\mathrm{ds}}=+10 \mathrm{~V}$ at time $=10 \mathrm{~ms}$, $\mathrm{a}-3.88-\mathrm{V} \Delta V_{\mathrm{th}}$ is observed. Here, the difference between the $\mathrm{HHI}$ erase and $-\mathrm{F}-\mathrm{N}$ methods is whether the drain voltage is applied. Therefore, the drain voltage effect on the memory erase characteristic is particularly investigated and shown in Fig. 6. While $V_{\mathrm{gs}}$ is fixed and $V_{\mathrm{ds}}$ is increased up to $10 \mathrm{~V}$, the device

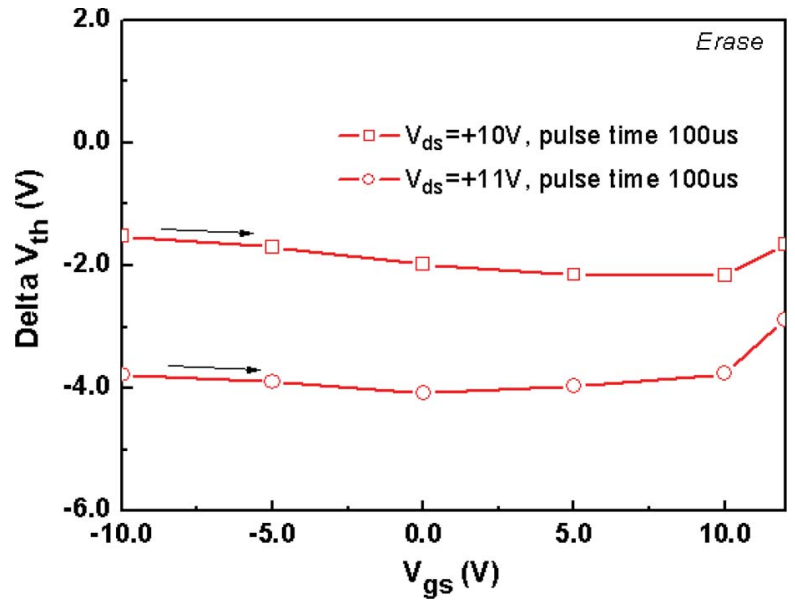

Fig. 7. Erased $\Delta V_{\mathrm{th}}$ as a function of different $V_{\mathrm{gs}}$ 's in the a-GIZO TFT NV memory.

shows normal CHE program characteristics, with $V_{\text {th }}$ positively shifting and the programmed window increasing well. However, once $V_{\mathrm{ds}}$ is greater than $10 \mathrm{~V}, V_{\text {th }}$ abruptly becomes negatively shifting and shows an erasing behavior. Furthermore, in Fig. 7, as the gate voltage is altered from $-10 \mathrm{~V}$ to $+12 \mathrm{~V}$, the negatively shifted $V_{\text {th }}$ almost stays at the same value level, whereas the drain voltage is set at 10 or $11 \mathrm{~V}$ for erasing the a-GIZO TFT NV memory. It means that, regardless of the polarity and magnitude of the gate voltage, erasing an a-GIZO TFT NV memory mainly depends on the level of drain voltage. It is different from the erasing characteristics of a Si MOSFET NV memory with a similar BB-HHI erase mode, which always needs $V_{\mathrm{gs}}<0$ and $V_{\mathrm{ds}}>\left|V_{\mathrm{gs}}\right|>0$ to attain the correct erasing characteristics.

These special erasing characteristics of the a-GIZO NV memory are thought to be the effect of one kind of avalanche BB-HHI stressing on a unique structure with wide material energy band. Similar to the n-type Si MOSFET NV memory, the large positive drain voltage lets the drain n-p junction to become reversely biased. While the energy band bending in the junction depletion region is higher than the semiconductor band gap, the direct tunneling of electrons from the conduct band minimum in the n-type semiconductor to the valence band maximum (VBM) in the p-type semiconductor becomes significant, and holes are then left on the VBM. Then, most electrons are collected by the drain terminal voltage, and some lucky holes get enough energy under the negative gate voltage to overcome the energy barrier limit between the channel and the tunneling insulator and to inject it into the charge trap layer. This neutralizes the trapped electrons in order to erase the programmed device. The huge tunneling electrical field is necessary for erasing the n-type Si MOSFET NV memory since the energy barrier of the hole-tunneling thin $\mathrm{SiO}_{2}$ layer from the p-type $\mathrm{Si}$ for charging the trap layer is $4.2 \mathrm{eV}$. The energy band structures of the a-GIZO (channel) $/ \mathrm{Al}_{2} \mathrm{O}_{3}$ (tunneling)/ a-GIZO (trapping) $/ \mathrm{Al}_{2} \mathrm{O}_{3}$ (blocking)/Mo (gate) system are depicted in Fig. 8(a). The energy barrier for an electron tunneling from the a-GIZO channel into the trapping layer is $3.0 \mathrm{eV}$, which is very close to the value of $3.15 \mathrm{eV}$ for a normal $\mathrm{Si} / \mathrm{SiO}_{2}$ system. In contrast, this hole barrier is only $2.0 \mathrm{eV}$ in 
Channel Tumneling Charge trap Blocking Bottom gate layer layer layer

a-GIZO $\mathrm{Al}_{2} \mathrm{O}_{3}$ a-GIZO $\mathrm{Al}_{2} \mathrm{O}_{3} \quad$ Mo

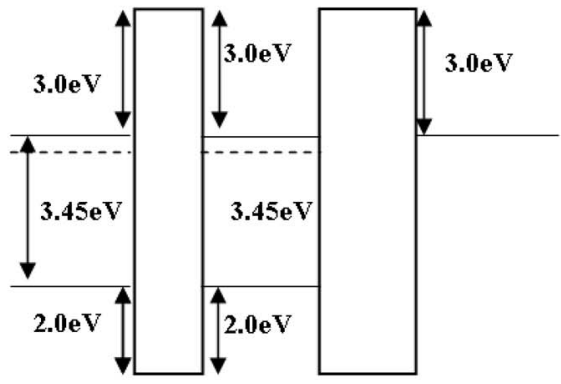

(a)

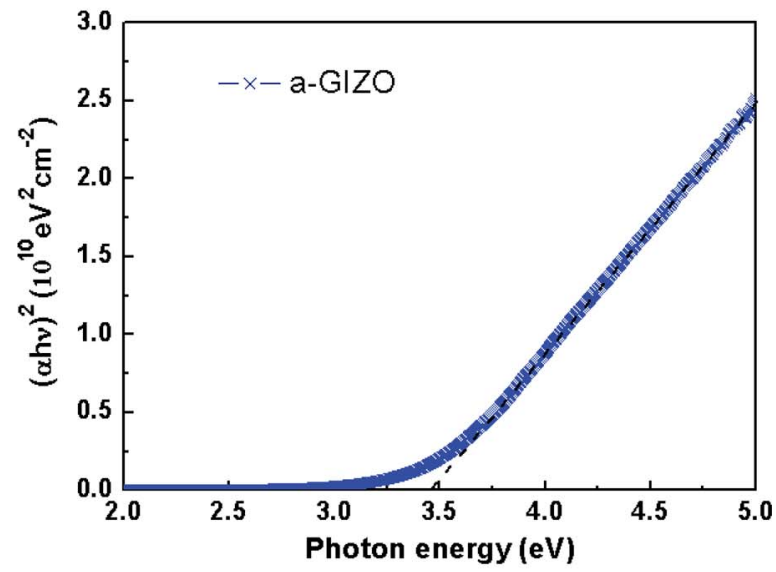

(b)

Fig. 8. Energy band structures of the a-GIZO (channel) $/ \mathrm{Al}_{2} \mathrm{O}_{3}$ (tunneling)/ a-GIZO (trapping) $/ \mathrm{Al}_{2} \mathrm{O}_{3}$ (blocking)/Mo (gate) system. (a) Energy band distribution level. (b) a-GIZO band gap estimated from the function of $(\alpha h v)^{2}$ versus $h v$ derivate from the measured optical absorption coefficient $\alpha$ gathered by the UV-visible spectrophotometer.

the a-GIZO TFT NV memory, which is much less than that of the $\mathrm{Si}_{-} \mathrm{SiO}_{2}$ system, because the a-GIZO has a wide-band-gap material with a value of approximately $3.45 \mathrm{eV}$. This band gap is estimated from the data gathered from the optical absorption coefficient $\alpha$ versus the wavelength plot by the UV-visible spectrophotometer in Fig. 8(b) [21].

In addition, unlike the homogeneous junction in $\mathrm{Si}$ MOSFET, the S/D contact with the channel in the a-GIZO TFT is considered as heterojunction, which is capable of being considered as the Schottky barrier diode or the ohmic contact [22] with different process conditions. As a result, the a-GIZO TFT can be treated as an n-type Schottky barrier transistor [23], because it shows similar transistor and diode $I-V$ characteristics. Therefore, the abrupt junction interface between the metal and the a-GIZO helps the occurrence of the carrier's band-to-band tunneling under a relatively low voltage. Hence, once the applied voltage is increased to some level $(\sim 10 \mathrm{~V})$, the electrons near junction tunneling into the drain electrode become depleted around the GIZO surface. The energy band of GIZO would gravely bend down to bring about hole accumulation around the surface for the charge balance. These holes are locally accelerated in the junction depletion region by a built-in electrical field or by the drain electrical field

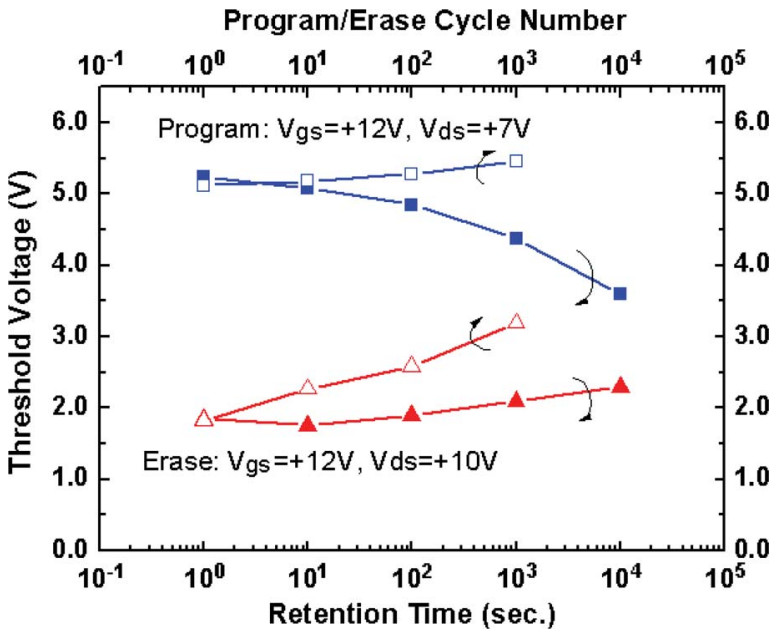

Fig. 9. P/E endurance and data retention characteristics of a-GIZO TFT NV memory.

since there is a long overlap length $(>2 \mu \mathrm{m})$ between the drain electrode and the gate electrode. Subsequently, a number of holes are injected into the gate insulator due to the small tunneling barrier $(\sim 2.0 \mathrm{eV})$ for the holes previously mentioned. For the hole-tunneling probability to surpass the electron-tunneling probability, the electrons (net negative charge) trapped in the trap layer are neutralized by the injected hole (positive charge), and the device's $V_{\text {th }}$ turns from positively shifting to negatively shifting. This speculation principally explained the unique erasing behavior of the a-GIZO NV memory decided by the drain voltage, regardless of the gate voltage. However, it needs quantitative work to confirm it in future.

The memory reliability characteristics, including P/E endurance and data retention characteristics, are shown in Fig. 9. In the figure, as the P/E cycles are greater than $10^{3}$ times or the retention time is more than $10^{3} \mathrm{~s}$, the programmed window decreased to be below $2.5 \mathrm{~V}$. The results indicate that the a-GIZO NV memory without postannealing does not have the same good reliability as a normal Si NV memory. The possible reason is believed to be that the a-GIZO film itself is sensitive to the atmosphere (especially, $\mathrm{O}_{2}$ ) and becomes unstable under long time exposure to air or electrical field stressing [18], [24]. The trapped charge in the trap layer easily escapes due to the film variation or is neutralized by the charges in the gate insulator and gate-insulator/channel interface. In addition, the accumulation of these charges in the gate insulator and interface also degrades the $\mathrm{P} / \mathrm{E}$ window after a number of cycles.

\section{Summary}

We demonstrated the possibility of producing NV memory by using an a-GIZO TFT as the driver and an a-GIZO as the charge trap layer under low process temperature. The memory program and, especially, the erase characteristics for the unique material structure are extensively investigated. The excellent $\mathrm{P} / \mathrm{E}$ characteristics of the $\mathrm{P} / \mathrm{E}$ window $>3.8 \mathrm{~V}$ at time $=10 \mathrm{~ms}$ on these oxide devices indicate that this technology is expected to be a promising choice in advanced SOP or 3-D stacking Flash memory products for the uniform film, low process temperature, and simple process steps. 


\section{ACKNOWLEDGMENT}

The authors would like to thank the staff of the NanoFabrication Center, Samsung Advanced Institute of Technology, for their support in the device fabrication.

\section{REFERENCES}

[1] S. B. Ogale, Thin Films and Heterostructures for Oxide Electronics. New York: Springer-Verlag, 2005.

[2] C.-U. Pinnow and T. Mikolajick, "Material aspects in emerging nonvolatile memories," J. Electrochem Soc., vol. 151, no. 6, pp. K13-K19, 2004.

[3] SIA. (2005, Dec.). ITRS 2005 Edition: Emerging Research Devices. [Online]. Available: http://www.itrs.net/Links/2005ITRS/ERD2005.pdf

[4] K. Kim, "Technology for sub-50 nm DRAM and NAND flash manufacturing," in IEDM Tech. Dig., 2005, pp. 323-326. session 13.4.

[5] I. G. Baek, M. S. Lee, S. Seo, M. J. Lee, D. H. Seo, D. S. Such, J. C. Park, S. O. Park, H. S. Kim, I. K. Yoo, U. I. Chung, and J. T. Moon, "Highly scalable non-volatile resistive memory using simple binary oxide driven by asymmetric unipolar voltage pulses," in IEDM Tech. Dig., 2004, pp. 587-590

[6] K. Szot, "Switching the electrical resistance of individual dislocations in single-crystalline $\mathrm{SrTiO}_{3}$," Nat. Mater, vol. 5, no. 4, pp. 312-320, Apr. 2006.

[7] Y. C. Chen, C. F. Chen, C. T. Chen, J. Y. Yu, S. Wu, S. L. Lung, R. Liu, and C. Y. Lu, "An access-transistor-free (0T/1R) non-volatile resistance random access memory (RRAM) using a novel threshold switching, selfrectifying chalcogenide device," in IEDM Tech. Dig., 2003, pp. 905-908.

[8] E. K. Lai, H. T. Lue, Y. H. Hsiao, J. Y. Hsieh, S. C. Lee, C. P. Lu, S. Y. Wang, L. W. Yang, K. C. Chen, J. Gong, K. Y. Hsieh, J. Ku, R. Liu, and C. Y. Lu, "A highly stackable thin-film transistor (TFT) NAND-type flash memory," in VLSI Symp. Tech. Dig., 2006, pp. 46-47. session 6.4 .

[9] H. J. Kim, "Uniform poly-Si TFTs for AMOLEDs using field-enhanced rapid thermal annealing," Solid State Phenom., vol. 124-126, pp. 447450, 2007.

[10] K. Nomura, H. Ohta, A. Takagi, T. Kamiya, M. Hirano, and H. Hosono, "Room-temperature fabrication of transparent flexible thin-film transistors using amorphous oxide semiconductors," Nature, vol. 432, no. 7016, pp. 488-492, Nov. 2004.

[11] H. Hosono, "Ionic amorphous oxide semiconductors: Material design, carrier transport, and device application," J. Non-Cryst. Solids, vol. 352, no. 9-20, pp. 851-858, Jun. 2006.

[12] R. E. Presley, "Transparent ring oscillator based on indium gallium oxide thin-film transistors," Solid State Electron., vol. 50, no. 3, pp. 500-503, Mar. 2006

[13] S. Ju, A. Facchetti, Y. Xuan, J. Liu, F. Ishikawa, P. Ye, C. Zhou, T. J. Marks, and D. B. Janes, "Fabrication of fully transparent nanowire transistors for transparent and flexible electronics," Nature Nanotechnol., vol. 2, pp. 378-384, 2007.

[14] H. Iechi, Y. Watanabe, and K. Kudo, "Inverter circuits using pentacene and ZnO transistors," Jpn. J. Appl. Phys., vol. 46, no. 4B, pp. 2645-2649, 2007.

[15] T. Nishibe and H. Nakamura, "Value-added circuit and function integration for SOG (system-on glass) based on LTPS technology," in Proc. SID, 2006, pp. 1091-1094.

[16] P. T. Liu, C. S. Huang, and C. W. Chen, "Enhanced planar poly-Si TFT EEPROM cell for system on panel applications," Electrochem. Solid-State Lett., vol. 10, no. 8, pp. J89-J91, 2007.

[17] C. J. Kim, D. Kang, I. Song, J. Park, H. Lim, S. Kim, E. Lee, R. Chung, J. Lee, and Y. Park, "Highly stable $\mathrm{Ga}_{2} \mathrm{O}_{3}-\mathrm{In}_{2} \mathrm{O}_{3}-\mathrm{ZnO}$ TFT for activematrix organic light-emitting diode display application," in IEDM Tech. Dig., 2006, pp. 307-310.

[18] D. Kang, H. Lim, C. J. Kim, I. Song, and J. G. Chung, "Amorphous gallium indium zinc oxide thin film transistors: Sensitive to oxygen molecules," Appl. Phys. Lett., vol. 90, no. 19, pp. 192 101-192 103, May 2007.

[19] P. Cappelletti, C. Golla, P. Olivo, and E. Zanoni, Flash Memories. Amsterdam, The Netherlands: Kluwer, 1999.

[20] S. Hsieh, H. Chen, Y. Chen, C. Chen, and Y. King, "MONOS memory in sequential laterally solidified low-temperature poly-Si TFTs," IEEE Electron Device Lett., vol. 27, no. 4, pp. 272-274, Apr. 2006.

[21] V. Srikant and D. R. Clarke, "On the optical band gap of zinc oxide," J. Appl. Phys., vol. 83, no. 10, pp. 5447-5451, May 1998.
[22] J. F. Wager, "Transparent electronics: Schottky barrier and heterojunction considerations," Thin Solid Films, vol. 516, no. 8, pp. 1755-1764, 2008. DOI: 10.1016/j.tsf.2007.06.164.

[23] S. Zhu, J. Chen, M.-F. Li, S. J. Lee, J. Sinfh, C. Z. Zhu, A. Du, C. H. Tung, A. Chin, and D. L. Kwong, "N-type Schottky barrier source/drain MOSFET using ytterbium silicide," IEEE Electron Device Lett., vol. 25, no. 8, pp. 565-567, Aug. 2004.

[24] P. Gorrn, P. Holzer, T. Riedl, W. Kowalsky, J. Wang, T. Weimann, P. Hinze, and S. Kipp, "Stability of transparent zinc tin oxide transistors under bias stress," Appl. Phys. Lett., vol. 90, no. 6, pp. 063 502-063 504, Feb. 2007.

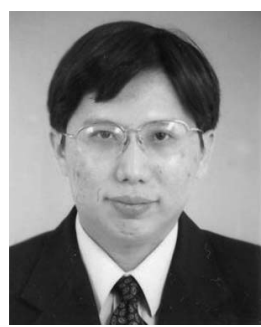

Huaxiang Yin (M’06) was born in Hunan, China. He received the B.S. degree in semiconductor physics and devices from Tianjin University, Tianjin, China, in 1996 and the M.S. and Ph.D. degrees in microelectronics and solid-state electronics from the Chinese Academy of Sciences, Beijing, China, in 1999 and 2003, respectively. During his studies, his research involved a series of process technology developments to deep sub-micro-CMOS ICs and, especially, an investigation of the design, modeling, and fabrication of CMOS FinFET structures on bulk

silicon substrate.

In 2003, he joined Samsung Advanced Institute of Technology, Yongin, Korea and is currently a Research Staff Member with the Semiconductor Device Laboratory. From 2003 to 2006, he worked on advanced low-temperature polysilicon TFT technologies for display on plastics, OLED driver, SOP, and new types of memory applications. He is the holder of tens of U.S. awarded and under awarding patents. He has authored the same number of technical papers. His current research interests include semiconducting oxide materials and device structures for novel memory applications.

Dr. Yin is a member of the IEEE Electron Devices Society.

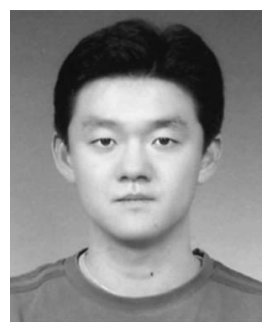

Sunil Kim received the B.S., M.S., and Ph.D. degrees in materials science and technology from Korea Advanced Institute of Science and Technology, Daejon, Korea, in 2000, 2001, and 2006, respectively. During his studies, he worked on the metallization process and epitaxial $\mathrm{CoSi}_{2}$ growth in silicon devices.

In 2006, he joined the Semiconductor Device Laboratory, Samsung Advanced Institute of Technology, Yongin, Korea. His current research interests include oxide semiconductor devices.

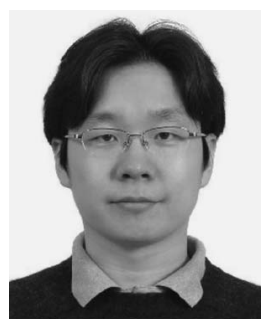

Hyuck Lim was born in Seoul, Korea, in 1976. He received B.S. and M.S. degrees from Seoul National University, Seoul, in 2000 and 2002, respectively. He is currently working toward the Ph.D. degree at the University of California San Diego, La Jolla, where he has been doing research on thermoelectricity.

From 2002 to 2003 , he worked on metal-induced lateral crystallization TFTs. In 2004, he joined the Semiconductor Device Laboratory, Samsung Advanced Institute of Technology, Yongin, Korea, where he studied low-temperature polysilicon TFT and GIZO TFT for flexible-display and transparent-display applications, respectively.

Yosep Min was with the Semiconductor Device Laboratory, Samsung Advanced Institute of Technology, Yongin, Korea 


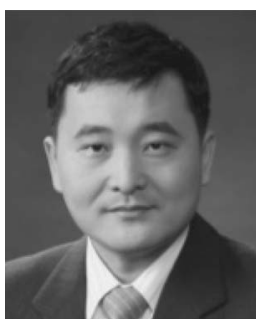

Chang Jung Kim was born in Seoul, Korea, on June 10, 1967. He received the B.S. degrees in inorganic materials engineering from the University of Hanyang, Seoul, in 1990 and the M.S. and Ph.D. degrees in materials science engineering from Korea Advanced Institute of Science and Technology, Daejon, Korea, in 1992 and 1995, respectively.

In 1995, he joined Samsung Advanced Institute of Technology, Yongin, Korea, where he has engaged in the research and development on ferroelectric nonvolatile memory devices. From 2002 to 2004, he was a Visiting Scholar with the University of Pennsylvania, Philadelphia, where he studied resistance memory devices. In 2005, he rejoined the Oxide Semiconductor TFT Project, Semiconductor Device Laboratory, Samsung Advanced Institute of Technology, Yongin, Korea, where he is currently working on $\mathrm{ZnO}$ related TFTs and oxide semiconductor devices. His research interests include the design and development of semiconductor thin films.

Dr. Kim is a member of the Electrochemical Society.

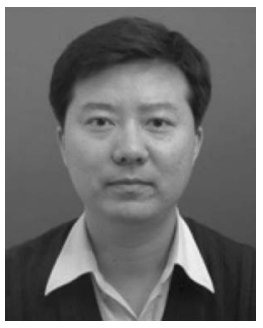

Ihun Song received the B.S., M.S., and Ph.D. degrees in applied physics from Sogang University, Seoul, Korea, in 1985, 1987, and 1998, respectively.

In 1987, he joined the Semiconductor Device Laboratory, Samsung Advanced Institute of Technology, Yongin, Korea, where he has engaged in the research and development of characterization of materials and fabrication processes of oxide semiconductor devices. He has also worked on fabrication processes, including MRAM, Josepson junction, and SQUID devices.

Dr. Song is a member of the Korean Physical Society.

Jaechul Park is with the Semiconductor Device Laboratory, Samsung Advanced Institute of Technology, Yongin, Korea.

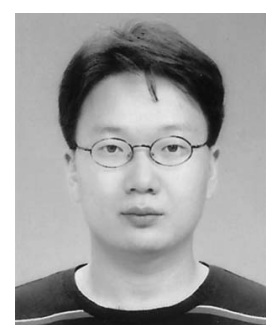

Sang-Wook Kim was born in Korea in 1978. He received the B.S. and M.S. degrees in materials science and engineering from Seoul National University, Seoul, Korea, in 2001 and 2003, respectively.

In 2006, he joined the Semiconductor Device Laboratory, Samsung Advanced Institute of Technology, Yongin, Korea, where he has been working on the research and development of oxide semiconductors.

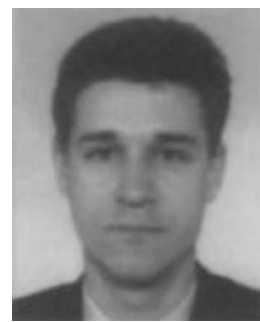

Alexander Tikhonovsky received the B.S. and M.S degrees in physics from the National Shevchenko University, Kiev, Ukraine, in 1994 and 1996, respectively, and the Ph.D. degree in crystalline materials science from Martin Luther University, Halle, Germany, in 2001.

In 2003, he joined the Analytical Engineering Center, Samsung Advanced Institute of Technology, Yongin, Korea, where he has been working on the research and development of advanced lowtemperature polysilicon TFTs and novel memory devices. He is currently with Samsung SDI Company, Ltd., Suwon, Korea.

Jaewoong Hyun is currently working toward the Ph.D. degree at the Harvard University, MA.

He was with the Semiconductor Device Laboratory, Samsung Advanced Institute of Technology, Yongin, Korea.

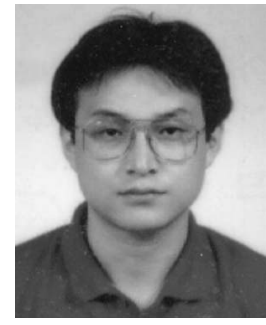

Youngsoo Park received the B.S. degree from Korea University, Seoul, Korea, in 1986 and the M.S. and $\mathrm{Ph} . \mathrm{D}$. degrees from Korea Advance Institute of Technology, Daejeon, Korea, in 1988 and 2000, respectively, all in materials science and engineering.

In 1991, he joined the Semiconductor Device Laboratory, Samsung Advanced Institute of Technology, Yongin, Korea, where he has been studying DLC films, diamond films, and poly-Si TFT devices. In 1995, he started FRAM research. He has joined an R\&D project on the world's first 1T-1C 64K FRAM, 4M FRAM, and 32M FRAM and is currently the Project Leader on the development of $\mathrm{ZnO}$ transistors for the application of OLED and resistive memory (ReRAM) for terabit density. 